DOI: 10.15587/2706-5448.2021.237269

Article type «Reports on Research Projects»

\section{Iryna Yaremchuk, Tetiana Bulavinets}

\title{
STUDY OF PLASMONIC PROPERTIES OF COPPER MONOSULFIDE NANOPARTICLES DEPENDING ON THEIR DIELECTRIC CONSTANT
}

The object of research is plasmonic properties copper of monosulfide nanoparticles. One of the most problematic areas is that there is still no unambiguous answer to which main copper monosulfide nanoparticles parameters have a decisive effect on their resonance absorption, scattering or electric field enhancement. It is necessary to study the plasmonic properties of copper monosulfide nanoparticles depending on their main parameter, namely the dielectric constant. The principle of dipole equivalence and Mee-Gans theory for the modeling of the optical nanoparticle characteristics is used. It is found that dielectric constant is a crucial parameter determining the resulting optical response of such nanoparticles. The surrounding medium refractive index affects the position and magnitude of the nanoparticles maximum plasmonic absorption. The nonspherical nanoparticles are characterized by two plasmon peaks corresponding to transverse and longitudinal localized surface plasmon resonance if the ratio between the axes is higher than 1.5. The ellipsoidal nanoparticles exhibit higher sensitivity to changes in the refractive index of the surrounding medium in comparison to the spherical ones. The obtained research results are primarily the basis for further comprehensive research of plasmonic copper monosulfide nanoparticles for their specialized applications. Second, knowledge of the influence of the nanoparticle dielectric constant on their resulting spectral characteristics allow tuning of the localized surface plasmon resonance peak position in a wide wavelength range, from 500 to $1200 \mathrm{~nm}$, using the nanoparticle synthesis technique. Thus, the material under study is promising for sensor applications in a wide spectral range.

Keywords: copper monosulfide, spherical and ellipsoidal nanoparticles, plasmon resonance peak, absorption cross section, dielectric constant.

\section{How to cite}

Yaremchuk, I., Bulavinets, T. (2021). Study of plasmonic properties of copper monosulfide nanoparticles depending on their dielectric constant. Technology Audit and Production Reserves, 4 (3 (60)), 9-13. doi: http://doi.org/10.15587/2706-5448.2021.237269

\section{Introduction}

In recent years copper sulfide nanoparticles have attracted considerable attention in terms of its use in various materials, optoelectronic devices, solar cells, sensors, and biomedicine [1]. This interest is primarily caused by low toxicity and high absorption coefficient in the near infrared region [2,3]. Copper monosulfide nanocrystals are characterized by strong near-infrared absorption because of localized surface plasmon resonance, which occurs because of free holes oscillations in the valence band of this material $[4,5]$. Variation in the composition and size of copper monosulfide nanoparticles allows one to change the width of the band gap and the concentration of free charge carriers. The intensity and position of the surface plasmon resonance maximum depends on the stoichiometry of copper monosulfide nanoparticles, morphology, and fabrication method. However, there is still no clear understanding of the nature of plasmon resonance [6]. The dielectric constant of $\mathrm{CuS}$ material depends on the synthesis technology. The change in the real and imaginary parts of the dielectric constant for polycrystalline $\mathrm{CuS}$ thin films depends on the copper salts from which they are synthesized. In [7] it was shown that the difference between the dielectric constants of four different $\mathrm{CuS}$ thin films is quite large: from 10 to 100 for the real part and from 0.25 to 4.0 for the imaginary part depending on the material composition and synthesis technology. In [8,9], the dielectric constants of copper monosulfide were calculated theoretically with the Drude-Sommerfeld theory and the results are also very different. Therefore, the technology of copper monosulfide synthesis will have a crucial influence on the optical response of such nanoparticles. Therefore, it is relevant to study the plasmonic properties of copper monosulfide nanoparticles depending on their main parameter, namely the dielectric constant. Thus, the object of our research is the plasmonic properties of copper monosulfide nanoparticles. The main aim is modeling of the absorption cross section spectra of the nanoparticles at the different copper monosulfide dielectric constants (synthesis conditions). The nanoparticles morphology and the refractive index of the surrounding medium were taking into account. 


\section{Methods of research}

The cross-sections of the optical absorption of spherical and ellipsoidal nanoparticles have been calculated during the study of plasmonic properties of copper monosulfide nanoparticles. The principle of dipole equivalence [10] was used in modeling the optical parameters of spherical nanoparticles, since the small size of nanoparticles (relative to the visible range wavelengths) allows confining by the dipole approximation. The Mi-Hans theory, which is based on different curvatures of the nanostructure surface and, thus, different reducing forces [11] was used to describe the optics of plasmon nanoparticles in the shape of ellipsoids of rotation.

In the calculations of the optical parameters of spherical nanoparticles, the light absorption cross section was determined by the value of electrostatic polarization $\alpha_{0}$. Electrostatic polarization in turn is determined by the wavelength-dependent optical dielectric constant $\varepsilon$. Therefore, nanospheres that located in the homogeneous dielectric medium $\left(\varepsilon_{m}\right)$, are described as:

$$
\alpha_{0}=\frac{3 V}{4 \pi} \frac{\varepsilon-\varepsilon_{m}}{\varepsilon+2 \varepsilon_{m}}=r^{3} \frac{\varepsilon-\varepsilon_{m}}{\varepsilon+2 \varepsilon_{m}},
$$

where $V$ - the nanoparticle volume; $r$ - the nanoparticle radius; $\varepsilon$ and $\varepsilon_{m}$ - dielectric functions of the nanoparticle material and the surrounding medium, respectively.

The extinction cross section of spherical nanoparticles is equal to the sum of the scattering and absorption cross sections and calculated by the ratio:

$$
C_{e x t}=C_{a b s}+C_{s c a}
$$

where absorption cross section $C_{a b s}$ describes the absorbed energy and is expressed in terms of the ratio of absorbed power to the energy flux in the incident light wave. The scattering cross section $C_{s c a}$ in turn describes that part of the energy flux that is scattered after interaction with the nanosphere and determined by the ratio of the scattered radiation power to the incident wave flux density.

$$
C_{a b s}=\frac{12 \pi k \varepsilon_{m} \operatorname{Im}\left(\varepsilon_{m}\right)}{r^{3}\left|\varepsilon-\varepsilon_{m}\right|^{2}}|\alpha|^{2}, \quad C_{s c a}=\frac{8 \pi}{3} k^{4}|\alpha|^{2},
$$

where the wave number of the nanoparticle surrounding medium is defined as:

$$
k=\frac{2 \pi \sqrt{\varepsilon_{h}}}{\lambda} .
$$

The renormalized polarization $\alpha$ is determined by the equation:

$$
\alpha=\frac{\alpha_{0}}{1+\varphi(k a) a^{-3} \alpha_{0}},
$$

where

$$
\varphi(k a)=2+2(i k a-1) \exp (i k a) \cong-(k a)^{2}-i \frac{2}{3}(k a)
$$

is a function describing radiation decay.
The optical parameters of nanoparticles in the shape of ellipsoid of rotation according to the Mi-Hans theory were calculated. The polarizations in the field parallel to the semiaxes $a, b$ and $c$ of the ellipsoid were calculated as follows:

$$
\begin{aligned}
& \alpha_{a}=4 \pi a b c \frac{\varepsilon-\varepsilon_{m}}{3 \varepsilon_{m}+3 L_{a}\left(\varepsilon-\varepsilon_{m}\right)}, \\
& \alpha_{b}=4 \pi a b c \frac{\varepsilon-\varepsilon_{m}}{3 \varepsilon_{m}+3 L_{b}\left(\varepsilon-\varepsilon_{m}\right)}, \\
& \alpha_{c}=4 \pi a b c \frac{\varepsilon-\varepsilon_{m}}{3 \varepsilon_{m}+3 L_{c}\left(\varepsilon-\varepsilon_{m}\right)} .
\end{aligned}
$$

where $L$ is geometric coefficient, defined as:

$$
\begin{aligned}
& L_{a}=\frac{a b c}{2} \int_{0}^{\infty} \frac{\mathrm{d} q}{\left(a^{2}+q\right) f(q)}, \\
& L_{b}=\frac{a b c}{2} \int_{0}^{\infty} \frac{\mathrm{d} q}{\left(b^{2}+q\right) f(q)}, \\
& L_{c}=\frac{a b c}{2} \int_{0}^{\infty} \frac{\mathrm{d} q}{\left(c^{2}+q\right) f(q)},
\end{aligned}
$$

where $f(q)=\sqrt{\left(q+a^{2}\right)\left(q+b^{2}\right)\left(q+c^{2}\right)}$.

It should be noted that only two of the three described geometric factors are independent, since $L_{a}+L_{b}+L_{c}=1$, and meet the condition $L_{a}<L_{b}<L_{c}$.

The used theoretical methods for studying the copper monosulfide nanoparticles optical parameters allow estimating the influence of size, shape, and parameters of surrounding medium on its optical response. In additional they should effectively tune the resonance of surface plasmons, shifting it to the desired spectral region.

\section{Research results and discussion}

The research of spectral characteristics of spherical copper monosulfide nanoparticles depending on the surrounding medium refractive index was carried out for four values of the copper monosulfide dielectric constant used from [7-9]. Nanoparticles with a radius of $30 \mathrm{~nm}$ were used for calculations in all four cases. The refractive index of the surrounding medium varied from 1.00 to 1.54 , namely $1.00 ; 1.33 ; 1.42$; 1.54 , which corresponds to the real values of such materials as air, water, muscle tissue and Canadian balm (resin/turpentine), which is used in dentistry. Such materials were chosen because copper monosulfide is characterized by plasmon absorption in the near infrared region, which, in turn, can be successfully used in various biomedical applications.

The dependence of the absorption cross section on the wavelength for copper monosulfide nanoparticles with dielectric constant used in [7] is shown in Fig. 1, $a$. As can be seen from this figure, spherical $\mathrm{CuS}$ nanoparticles in the air have an absorption peak at the wavelength of $0.455 \mu \mathrm{m}$. The absorption cross section spectrum is characterized by the peak's full width at half maximum of about $90 \mathrm{~nm}$. The position of the plasmon absorption peak shifts to the longer wavelength's region and the full width at half maximum of the spectrum increases when the refractive index of the surrounding medium changes. The absorption peak of nanoparticles located in the medium with a refractive index 
of 1.54 is at a wavelength of 0.672 and the full width at half maximum equal to 0.166 . However, it should be noted that the amplitude of the spectrum practically does not change.

The dependence of the absorption cross section on the wavelength of copper monosulfide nanoparticles with experimentally determined dielectric constant (in [8] Fig. 5, curve $d$ ) is shown in Fig. $1, b$. This figure shows that the plasmon resonance peak is in the visible spectral region for all refractive indices of the surrounding medium and it is about $0.6 \mu \mathrm{m}$. The absorption peak is practically not shifted at changing the refractive index of surrounding medium. However, the value of the absorption cross section at the resonant wavelength increases with the increasing of surrounding medium refractive index. Such position of plasmon absorption as in these two described cases is typical for pure copper nanoparticles. Therefore, let's assume that the synthesized copper monosulfide contains a significant proportion of $\mathrm{Cu}$.

The dependence of the absorption cross section on the wavelength for copper monosulfide nanoparticles with dielectric constant used in [8, Fig. 5, curve a] is shown in Fig. 1, c. As can be seen from the figure, such spherical $\mathrm{CuS}$ nanoparticles are not characterized by a pronounced absorption peak. A small absorption peak at a wavelength of about $1.05 \mu \mathrm{m}$ is visible. The behavior of the absorption peak does not change with increasing of surrounding medium refractive index.

A completely different picture is observed for $\mathrm{CuS}$ nanoparticles with a refractive index calculated according to Drude-Sommerfeld theory presented in [9] (Fig. 1, d).
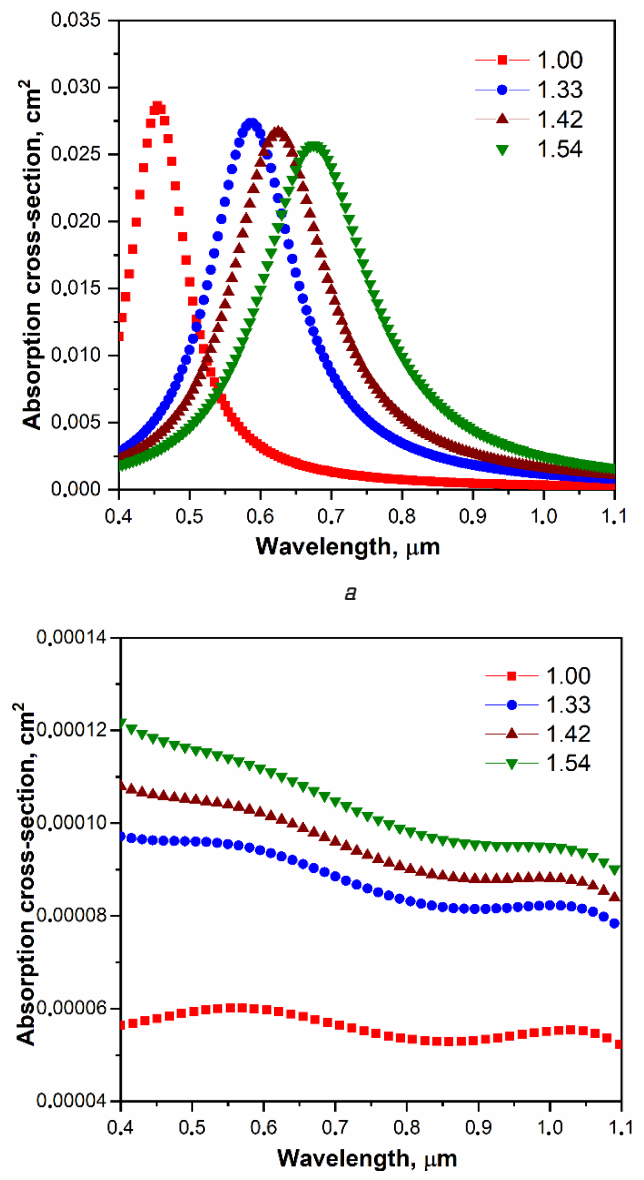

c
The figure shows a clear absorption cross section peak at a wavelength of $0.93 \mu \mathrm{m}$ for nanoparticles located in the air. As the surrounding medium refractive index increases, the plasmon absorption peak shifts to the longer wavelength's region. For example, the peak of the plasmon resonance is at a wavelength of $1.06 \mu \mathrm{m}$ for the value of surrounding medium refractive index equal to 1.54 .

In addition, as the surrounding medium refractive index increases, the maximum value of the absorption cross section increases from 0.0046 to $0.0101 \mathrm{~cm}^{2}$ at the resonant wavelength. Such nanoparticles can be successfully used in the near-infrared region in a «second biological window» [12].

Therefore, the critical parameter that determines the resulting optical response of copper monosulfide nanoparticles is their dielectric constant, which is determined by the synthesis conditions. Besides, the surrounding medium refractive index will affect the position and magnitude of the maximum absorption cross-section of nanoparticles.

During the synthesis of nanoparticles, it is not always possible to obtain nanoparticles of perfectly spherical shape, thus at the next stage of work the modeling of nanoparticles in the shape of ellipsoids was carried out. The calculations were limited to only one value of the complex dielectric constant of copper monosulfide, namely, calculated from the data presented in [11]. The dependence of the absorption cross section on the wavelength of nonspherical $\mathrm{CuS}$ nanoparticles at different values of the ratio between the axes $(e)$ is presented in Fig. 2, $a$.

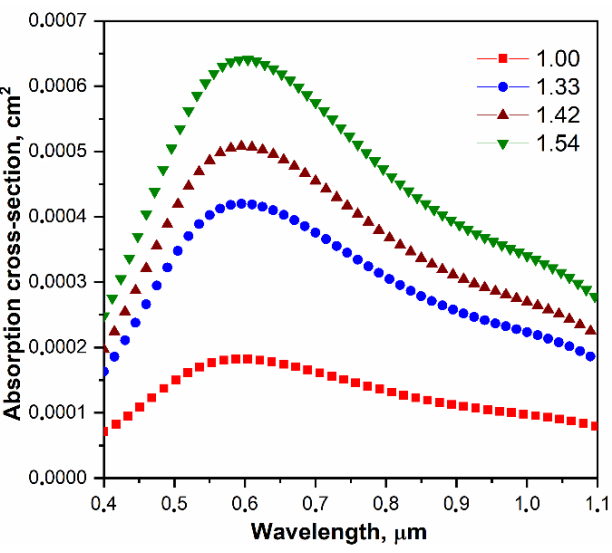

$b$

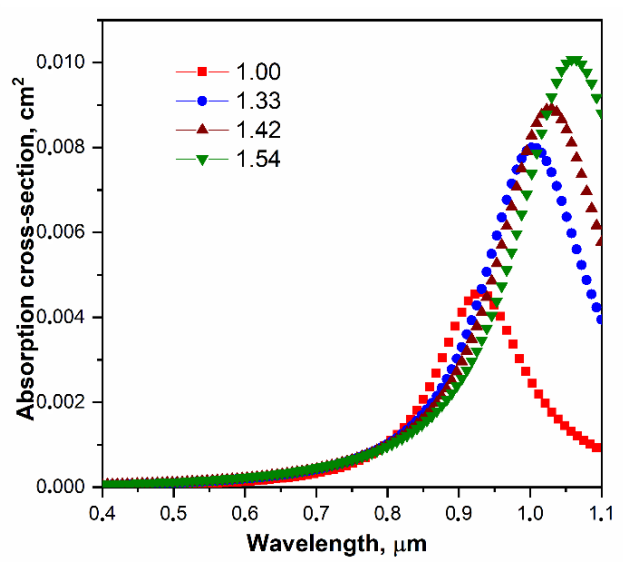

d

Fig. 1. Absorption cross sections spectra of spherical copper monosulfide nanoparticles at the different values of the surrounding medium refractive index. The data of dielectric constant of copper monosulfide was used from: $a-$ ref. [7]; $b$ - ref. [8, Fig. 5, curve d]; $c-r e f . ~[8$, Fig. 5, curve a]; $d-$ ref. [9] 


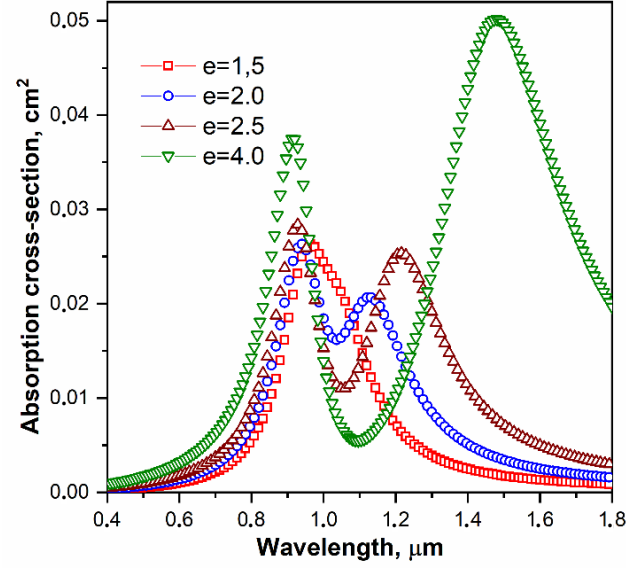

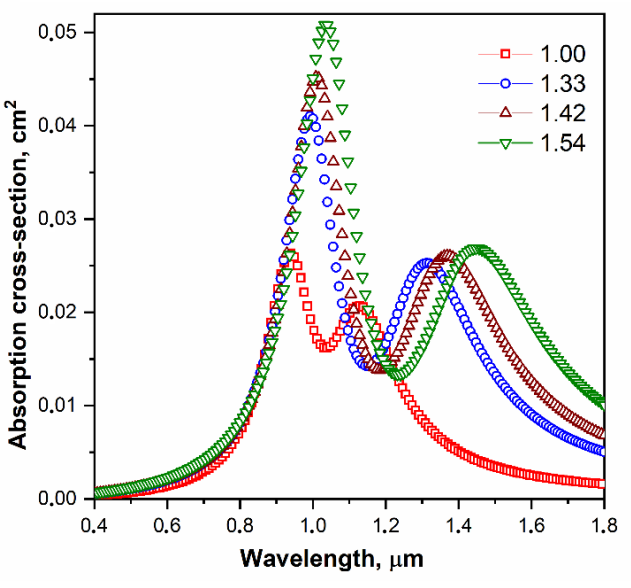

b

Fig. 2. Absorption cross sections spectra of ellipsoidal copper monosulfide nanoparticles:

$a$ - at the different values of the ratio between axes; $b$ - at the different values of the surrounding medium refractive index

The shape of the nanoparticles was chosen that one semiaxis was changed and the other two remained constant. The semiaxis $a$ varied from $15 \mathrm{~nm}$ to $40 \mathrm{~nm}$, the axes $b$ and $c$ were chosen to be equal to $10 \mathrm{~nm}$. The figure clearly shows that nonspherical nanoparticles are characterized by two plasmon peaks, which corresponds to the transverse and longitudinal localized surface plasmon resonance if the ratio between the axes is more than 1.5. As the ratio between the axes increases, the maximum of the second peak and the width between the peaks increase. It should be noted that the position of the first maximum remains constant when the size of the nanoparticle changes, and the position of the second maximum shifts to the long-wavelength spectral region. The influence of the surrounding medium refractive index on the resulting absorption cross section was studied for this type of nanoparticles (Fig. 2, $b$ ).

It can be seen from Fig. 2, $b$ that the first peak is less sensitive to changes in the surrounding medium dielectric constant than the second one, namely the first peak is shifted by $0.097 \mu \mathrm{m}$, and the second by $0.32 \mu \mathrm{m}$ when the refractive index changes from 1.0 to 1.54 . A comparison of Fig. 1, $d, 2, b$ shows that spheroidal nanoparticles demonstrate a higher sensitivity to changes in the surrounding medium refractive index.

However, the limitation of this all study is that the chosen research technique does not allow calculating the distribution of the electromagnetic field in the copper monosulfide nanoparticles under localized surface plasmon resonance conditions. As it is known, such knowledge is necessary at designing the real structures for their application in sensors, SERS or photovoltaics. In addition, it should be noted that the main disadvantage of this study is not taking into account specific parameters that affect the value of the dielectric constant. Thus, the plasma frequency, concentration of free charge carriers and damping constant of free carriers must be taken into account for the synthesis of copper sulfide nanoparticles with the given dielectric constant.

\section{Conclusions}

It is shown that the method of nanostructured copper monosulfide manufacture will be crucial for their resulting characteristics, namely the position of the plasmon resonance peak. The dependences of the absorption cross section on the wavelength of spherical copper monosulfide nanoparticles at different surrounding medium refractive indices have been studied. The surrounding medium refractive index primarily affects the amplitude of the dependence of absorption cross section on the wavelength. In addition, it affects the plasmon absorption peak position. The peak of the plasmon resonance is shifted from 0.93 to $1.06 \mu \mathrm{m}$ when the surrounding medium refractive index changes from 1.00 to 1.54 . The dependences of the absorption cross section on the wavelength of nonspherical sulfide nanoparticles were calculated. It is shown that ellipsoidal copper monosulfide nanoparticles are characterized by the two peaks of plasmon resonance. Moreover, the value of the maximum of the first peak remains constant, and the value of the second peak increases with increasing nanoparticle size, namely the first peak is shifted by $0.097 \mu \mathrm{m}$, and the second one by $0.32 \mu \mathrm{m}$ when the refractive index changes from 1.0 to 1.54 . The distance between the peaks will depend on the ratio between the axes of nonspherical nanoparticle, it is $0.176 \mu \mathrm{m}$ for $e=2.0$ and $0.573 \mu \mathrm{m}$ for $e=4.0$.

The obtained research results show possibility to control of the plasmonic properties of the copper monosulfide nanoparticles in a wide wavelength range using the nanoparticle synthesis technique. Knowledges of influence dielectric constant on resulting optical response allows obtaining of the nanoparticles with given parameters for different applications.

\section{Acknowledgements}

The authors acknowledge the financial support of this research by the Ministry of Education and Science of Ukraine under the grant «Nanostructured interfaces based on nontoxic materials for engineering applications» (DB/Interface no. 0120u100675).

\section{References}

1. Goel, S., Chen, F., Cai, W. (2013). Synthesis and Biomedical Applications of Copper Sulfide Nanoparticles: From Sensors to Theranostics. Small, 10 (4), 631-645. doi: http://doi.org/ 10.1002/smll.201301174 
2. Huang, J., Zhou, J., Zhuang, J., Gao, H., Huang, D., Wang, L. et. al. (2017). Strong Near-Infrared Absorbing and Biocompatible CuS Nanoparticles for Rapid and Efficient Photothermal Ablation of Gram-Positive and -Negative Bacteria. ACS Applied Materials E Interfaces, 9 (42), 36606-36614. doi: http://doi.org/ 10.1021/acsami.7b11062

3. Zhang, Q., Jia, G., Zhang, W., Zhao, Z. (2021). Infrared plasma photothermal conversion of $\mathrm{Cu} 2-\mathrm{xS} /$ cellulose nanofilms prepared by sequential reaction. Results in Physics, 22, 103942. doi: http://doi.org/10.1016/j.rinp.2021.103942

4. Homola, J., Yee, S. S., Gauglitz, G. (1999). Surface plasmon resonance sensors: review. Sensors and Actuators B: Chemical, 54 (1-2), 3-15. doi: http://doi.org/10.1016/s0925-4005(98)00321-9

5. Xie, Y., Chen, W., Bertoni, G., Kriegel, I., Xiong, M., Li, N. et. al. (2017). Tuning and Locking the Localized Surface Plasmon Resonances of $\mathrm{CuS}$ (Covellite) Nanocrystals by an Amorphous CuPdxS Shell. Chemistry of Materials, 29 (4), 1716-1723. doi: http://doi.org/10.1021/acs.chemmater.6b05184

6. Lian, Z., Sakamoto, M., Matsunaga, H., Vequizo, J. J. M., Yamakata, A., Haruta, M. et. al. (2018). Near infrared light induced plasmonic hot hole transfer at a nano-heterointerface. Nature Communications, 9 (1). doi: http://doi.org/10.1038/ s41467-018-04630-w

7. Erken, O., Gunes, M., Kirmizigul, F., Gumus, C. (2018). Investigation of properties the copper sulfide thin films prepared from different copper salts. Optik, 168, 884-891. doi: http:// doi.org/10.1016/j.ijleo.2018.05.031

8. Córdova-Castro, R. M., Casavola, M., van Schilfgaarde, M., Krasavin, A. V., Green, M. A., Richards, D., Zayats, A. V (2019). Anisotropic Plasmonic CuS Nanocrystals as a Natural
Electronic Material with Hyperbolic Optical Dispersion. ACS Nano, 13 (6), 6550-6560. doi: http://doi.org/10.1021/acsnano. $9 \mathrm{~b} 00282$

9. Lesyuk, R., Klein, E., Yaremchuk, I., Klinke, C. (2018). Copper sulfide nanosheets with shape-tunable plasmonic properties in the NIR region. Nanoscale, 10 (44), 20640-20651. doi: http:// doi.org/10.1039/c8nr06738d

10. Khlebtsov, N. G. (2008). Optics and biophotonics of nanoparticles with a plasmon resonance. Quantum Electronics, 38 (6), 504-529. doi: http://doi.org/10.1070/qe2008v038n06abeh013829

11. Prescott, S. W., Mulvaney, P. (2006). Gold nanorod extinction spectra. Journal of Applied Physics, 99 (12), 123504. doi: http://doi.org/10.1063/1.2203212

12. Liu, Y., Gao, D., Xu, M., Yuan, Z. (2018). Multispectral photoacoustic imaging of cancer with broadband $\mathrm{CuS}$ nanoparticles covering both near-infrared I and II biological windows. Journal of Biophotonics, 12 (3). doi: http://doi.org/10.1002/jbio.201800237

$\triangle$ Iryna Yaremchuk, Doctor of Technical Sciences, Professor, Associate Professor, Department of Photonics, Lviv Polytechnic National University, Lviv, Ukraine, ORCID: https://orcid.org/0000-00027072-5950, e-mail: iryna.y.yaremchuk@lpnu.ua

Tetiana Bulavinets, PhD, Assistant, Department of Photonics, Lviv Polytechnic National University, Lviv, Ukraine, ORCID: https:// orcid.org/0000-0001-6898-3363

$\triangle$ Corresponding author 